\title{
Agaricales del Chaco Oriental 1. Primer Registro de TETRAPYRgos NIGRIPES (MARAsMIACEAE) Y XEROMPHALINA TENUIPES (MYCENACEAE) PARA LA REgIÓN
}

\author{
NATALIA A. RAMÍREZ, NICOLÁS NIVEIRO y ORLANDO F. POPOFF ${ }^{1}$
}

\begin{abstract}
Summary: Agaricales from the eastern Chaco 1: First record of Tetrapyrgos nigripes (Marasmiaceae) and Xeromphalina tenuipes (Mycenaceae) for the region. Tetrapyrgos nigripes and Xeromphalina tenuipes are described and illustrated, constituting first records of these two pantropical species for the Chaco Oriental region. Tetrapyrgos nigripes is characterized by its centrally stipitated pileus with tetrahedric spores and a pileipellis with rameal structures, while $X$. tenuipes has a yellowish to ochraceus orange pileus, paler yellow lamellae, and a reddish brown stipe covered by abundant hairs that give it a velvety appearance.
\end{abstract}

Key words: Basidiomycota, lignicolous fungi, Colonia Benitez Natural Educative Reserve, diversity.

\begin{abstract}
Resumen: Se describen e ilustran a Tetrapyrgos nigripes y Xeromphalina tenuipes, registros nuevos para la región del Chaco Oriental, de estas dos especies pantropicales. Tetrapyrgos nigripes se caracteriza por su píleo centralmente estipitado con esporas tetraédricas y pileipellis con estructuras rameales, mientras que $X$. tenuipes presenta el píleo amarillento a ocráceo anaranjado, laminillas amarillas más claras y estípite marrón rojizo cubierto por abundantes pelos que le dan un aspecto aterciopelado.
\end{abstract}

Palabras clave: Basidiomycota, hongos lignícolas, Reserva Natural Educativa Colonia Benítez, diversidad.

\section{INTRODUCCIÓN}

La subregión del Chaco Oriental (Cabrera, 1994) o Chaco Húmedo (Burkart et al., 1999) en el norte de Argentina consiste en una franja más o menos estrecha que se extiende paralelamente a los ejes de los ríos Paraná y Paraguay, al este de las provincias de Formosa y Chaco, noreste de Santa Fe y oeste de Corrientes. Su vegetación es muy heterogénea, presentándose como un mosaico complejo donde los distintos tipos de bosque están dispersos formando isletas sobre una matriz de pastizales, pajonales y humedales (Maturo et al., 2005; Morello \& Adamoli, 1967) y ha sido caracterizada como transicional entre el Dominio Chaqueño y Amazónico (Prado, 1993). Actualmente, en la región del Chaco Oriental,

\footnotetext{
1 Laboratorio de Micología, Instituto de Botánica del Nordeste (UNNE-CONICET), Sgto. Cabral 2131, C.C. 209, C.P. 3400, Corrientes, Argentina. nataliaandrearamirez@hotmail.com.ar - niconiveiro@ hotmail.com
}

se considera la coexistencia de dos Dominios Fitogeográficos: los Bosques Secos Estacionales Neotropicales (BSEN) (Prado, 2000) y los Bosques Chaqueños (Cabrera, 1994). Los BSEN están representados por los Bosques Transicionales AustroBrasileños (Prado, 1993) a los que pertenecen los Bosques Ribereños (Maturo et al., 2005, 2012); y el Dominio Chaqueño, se encuentra representado por los bosques dominados por Schinopsis balansae. En dicha región se encuentra ubicada la Reserva Educativa "Colonia Benítez", que cuenta con un poco más de 7 ha de superficie. El área se encuentra en un relicto del antiguo cauce del río Tragadero y a pesar de su escaso tamaño, presenta las comunidades vegetales características del Chaco Oriental bien definidas (Lanfiutti, 1998).

Los hongos xilófagos revisten de gran importancia en los ecosistemas boscosos debido a su actividad sapróbica como descomponedores. Estos reciclan las sustancias lignocelulósicas reincorporándolas al ecosistema (Popoff \& Ferraro, 2002; Webster \& Weber, 2007). Además, su actividad degradadora 
debilita la madera facilitando a aves y pequeños mamíferos la realización de sus nidos o refugios, como así también son utilizados por diferentes invertebrados como alimento y hábitat (Lodge et al., 2004).

Los estudios realizados sobre Agaricales en la región del Chaco Oriental son muy escasos. Spegazzini, a finales del siglo XIX, realizo varias colecciones en la zona de estudio (Spegazzini, 1899), y desde ese trabajo solo se cuenta con los estudios actuales para la región (Popoff, 2000; Niveiro et al., 2009a-b, 2010). Niveiro \& Popoff (2010a) citan 79 taxones de hongos para el Distrito Chaqueño Oriental, de los cuales solo 28 han sido coleccionados en la Reserva Educativa Colonia Benítez (Niveiro \& Popoff, 2009).

Debido a que se cuenta con escasa información de los agaricales en esta región, el objetivo del presente trabajo es dar a conocer dos nuevas citas para la provincia del Chaco, describiendo, ilustrando y discutiendo la taxonomía de las especies colectadas.

\section{Materiales y Métodos}

El material coleccionado fue fotografiado y descrito macroscópicamente in situ, utilizando la terminología propuesta por Largent (1986) y Lodge et al. (2004). Para las observaciones microscópicas (elementos del revestimiento piléico, del contexto del píleo y del pie, de las laminillas, esporas, basidios, cistidios, etc.) se realizaron cortes a mano alzada montados en $\mathrm{KOH}$ 5\% teñidos con floxina acuosa al $1 \%$, y reactivo de Melzer (Wright \& Albertó, 2002).

Los autores de los nombres científicos se indican de acuerdo a Index Fungorum - Authors of Fungal Names (2012), las siglas de los herbarios según Thiers (2012). El material recogido fue depositado en el herbario del Instituto de Botánica del Nordeste (CTES).

\section{Resultados}

Tetrapyrgos nigripes (Schwein.) E. Horak. Sydowia 39: 102. 1987 [1986]. (Tipo no examinado), (Fig. 1A-C).

$\equiv$ Agaricus nigripes Schwein., Schr. Naturf. Ges. Leipzig 1: 678. 1822. $\equiv$ Marasmiellus nigripes (Schwein.) Singer, Pap. Mich. Acad. Sci. 32(1): 130. 1946 [1948].

$\equiv$ Pterospora nigripes (Schwein.) E. Horak, Sydowia 36: 133. 1983.

= Marasmius dichromopus Speg., Bol. Acad. Nac. Ci. 28: 293. 1926.

Píleo 10-25 mm de diám., convexo campanulado a plano convexo, centro a veces depreso hasta umbilicado, superficie lisa, húmeda, blanca, volviéndose grisácea verdosa en los márgenes a gris oscuro o gris amarronado en el centro, tornándose verdoso a azul verdoso al tacto, margen entero, translúcidamente estriado a sulcado (Fig. 1A). Contexto delgado, blanquecino, oscureciéndose al exponerse. Olor y sabor no testeados. Laminillas regulares o con intervenosas cortas, sobre todo en ejemplares maduros, blanquecinas, próximas, adnatas a subdecurrentes, margen entero, concoloro, con lamélulas. Pie central, 11-32 x 1-2 mm, cilíndrico, igual, gris oscuro, más claro a blanco hacia la base, pruinoso, hueco, de consistencia cartilaginosa. Esporada blanca.

Esporas 9-10 x 5-9 $\mu \mathrm{m}, x=8,85 \times 7,02 \mu \mathrm{m}$; $\mathrm{Q}=$ $1,02-2,00 ; \mathrm{Q}_{\mathrm{x}}=1,29 ; \mathrm{n}=17 ; \mathrm{N}=2$; tetraédricas, lisas, hialinas, inamiloideas, con paredes delgadas, sin poro germinativo (Fig. 1B). Basidios 33-40 × 7-9 $\mu \mathrm{m}$, claviformes, tetrasporados. Pleurocistidios ausentes. Queilocistidios 22-37 x 7-11 $\mu \mathrm{m}$, cilíndricos, con diversas ramificaciones hacia el extremo distal, con procesos digitiformes, hialinos, cubiertos por abundantes excrecencias simples, cilíndricas (Fig. 1C). Trama himenoforal subregular a irregular, con hifas hialinas de 4-9 $\mu \mathrm{m}$ de diám. Pileipellis formada por hifas diverticuladas y estructuras rameales, 10-25 x 7-13 $\mu \mathrm{m}$. Fíbulas presentes.

Hábito y hábitat. Solitarios o gregarios, connados, cespitosos, xilófagos, sobre ramas caídas semienterradas y raíces.

Material examinado. ARGENTINA, Prov. Chaco, Dpto. $1^{\circ}$ de Mayo, Reserva Educativa Colonia Benítez, en sendero interpretativo, sobre ramas de dicotiledóneas no identificadas, $\left(27^{\circ} 19^{\prime}\right.$ 04,12" S $58^{\circ} 56^{\prime} 59,58^{\prime \prime} \mathrm{W}, 64 \mathrm{msnm}$.), 18/X/2008, leg. N. Ramírez et al. 17 y 32 (CTES).

Obs. el género Tetrapyrgos se distingue fácilmente por sus esporas tetraédricas combinadas 


\section{N. A. Ramírez et al. - Tetrapygros nigripes y Xermophalina tenuipes en el Chaco Oriental}

con la pileipellis con estructuras rameales. Horak (1986) propone este género, combinando a Marasmiellus nigripes, con varias especies que se encontraban incluidas en Campanella, género con especies de hábito pleurotoides (Singer, 1986), por lo que M. nigripes es la única especie centralmente estipitada del género.

Distribución. Especie ampliamente distribuida en los trópicos (Pegler, 1986). En la Argentina fue citada para las provincias de Salta (Singer, 1973), Tucumán, como Lentinus nigripes (Spegazzini, 1919), Misiones (Wright \& Wright, 2005) y Buenos Aires (Singer, 1973; Raithelhuber, 2004).

Xeromphalina tenuipes (Schwein.) A. H. Sm. Pap. Mich. Acad. Sci. 38: 84, 1953. (Tipo no examinado), (Fig. 2 A-D).

$\equiv$ Agaricus tenuipes Schwein., Trans. Amer. Philos. Soc. 4(2): 147. 1832.

$\equiv$ Heimiomyces tenuipes (Schwein.) Singer, Mycologia 35(2): 159. 1943.

= Heliomyces rheicolor (Berk.) Speg., Bol. Acad. Nac. Ci. 23(3-4): 386. 1919.

= Agaricus (Collybia) aurantiellus Speg., Anales Soc. Ci. Argent. 16(5): 244. 1883.

$=$ Collybia aurantiella (Speg.) Speg., Anales Soc. Ci. Argent. 25: 221. 1887.

Píleo 10-70 mm. diám., convexo a plano convexo, finalmente anchamemte umbonado, a veces levemente depreso con la madurez, margen recurvado, translúcidamente sulcado a estriado, superficie seca, macroscópicamente lisa, pubescente, velutina a granulosa bajo lupa, glabrescente, higrófano, amarillo a rojo amarronado, volviéndose ocráceo anaranjado, margen generalmente mas claro, a veces completamente amarillo (Fig. 2A). Contexto delgado, crémeo. Olor y sabor no testeados. Himenóforo lamelado. Laminillas con intervenosas, crémeas a amarillentas, próximas, anexas a adnatas, margen entero, concoloro con los lados, con lamélulas, intervenosas bajas que no superan la mitad de la laminilla. Pie central, 15-65 x 2-4 mm, cilíndrico a aplanado, a veces con un surco longitudinal, cilíndrico o adelgazándose hacia el ápice, anaranjado a anaranjado amarillento, oscureciéndose hacia la base, marcadamente piloso, a veces con una pseudoriza larga. Esporada blanca.

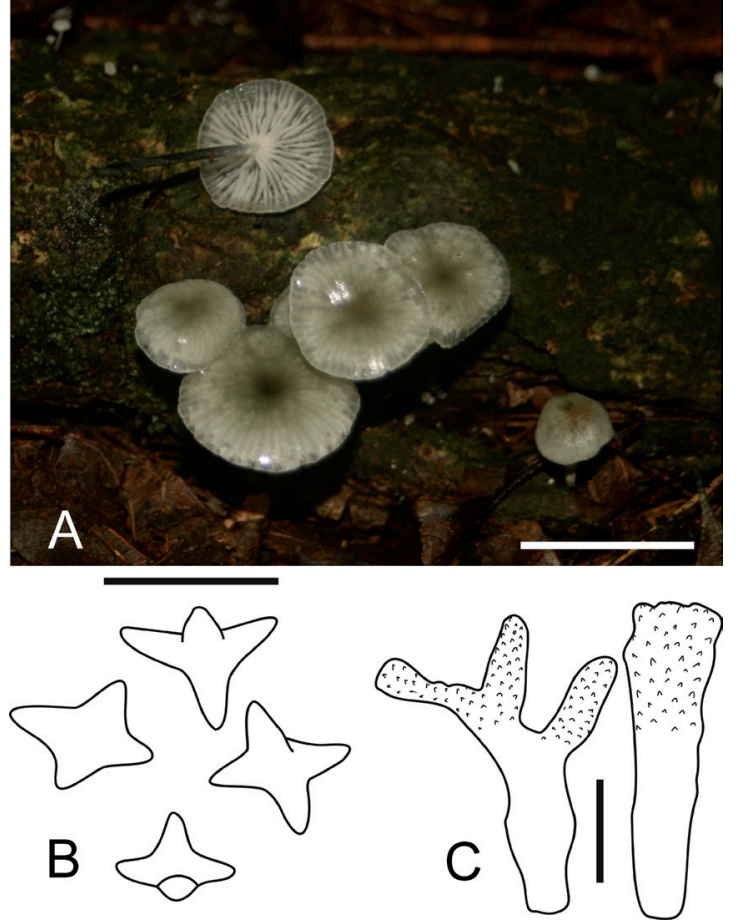

Fig. 1. Tetrapyrgos nigripes. A: Basidiomas. B: Esporas. C: Queilocistidios. Escalas: $A=20 \mathrm{~mm}$; B y $C=10 \mu \mathrm{m}$.

Esporas 5,9-8,4 x 2,9-4,3 $\mu \mathrm{m} ; x=7,2 \times 3,6 \mu \mathrm{m}$; $\mathrm{Q}=1,55-2,58 ; \mathrm{Q}_{\mathrm{x}}=1,98 ; \mathrm{n}=20 ; \mathrm{N}=2$; elipsoidales a subcilíndricas, con paredes delgadas, lisas, hialinas, amiloides, sin poro germinativo (Fig. 2B). Basidios 29-37 x 5,7-8,9 $\mu$ m., claviformes, tetraesporados, de paredes delgadas, lisas (Fig. 2C). Basidiolos 22-28 x 3,8-5,8 $\mu \mathrm{m}$., claviformes a fusiformes, de paredes delgadas, lisas, muy abundantes. Pleurocistidios ausentes. Queilocistidios 22-35 x 2,5-5 $\mu \mathrm{m}$., delgadamente claviformes a cilíndricos, sinuosos, hialinos. Trama himenoforal irregular, hifas de 1,5-4 $\mu \mathrm{m}$. de diám., inamiloides, con paredes gelatinosas. Pileipellis en un epicutis formado por una empalizada irregular de pelos setiformes (Fig. 2D), erectos o semierectos, 50-220 x 5-18 $\mu \mathrm{m}$, cilíndricos, con ápices redondeados, de paredes engrosadas, ferrugíneas. Caulocistidios semejantes a los pelos setiformes de la pileipellis. Fíbulas presentes.

Hábito y hábitat. Solitarios o gregarios, xilófagos, sobre tocones o ramas caídas semienterradas. 
Bol. Soc. Argent. Bot. 48 (3-4) 2013

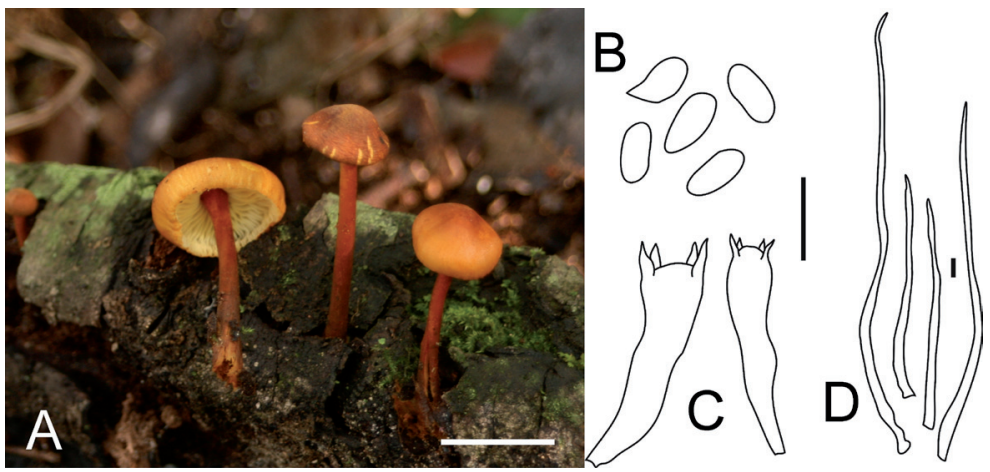

Fig. 2. Xeromphalina tenuipes. A: Basidiomas. B: Esporas. C: Basidios. D: Pelos setiformes de la pileipellis. Escalas: $A=20 \mathrm{~mm}$; $B, C$ y $D=10 \mu \mathrm{m}$.

Material examinado. ARGENTINA, Prov. Chaco, Dpto. $1^{\circ}$ de Mayo, Reserva Educativa Colonia Benítez, en sendero interpretativo, sobre ramas de dicotiledóneas no identificadas, $\left(27^{\circ}\right.$ $19^{\prime} 04,12^{\prime \prime} \mathrm{S} 58^{\circ} 56^{\prime} 59,58^{\prime \prime} \mathrm{W}, 64 \mathrm{msnm}$.), $18 / \mathrm{X} / 2008$, leg. N. Niveiro 849, 869 y 870 (CTES).

Obs. Especie pantropical, que se caracteriza por sus colores llamativos, píleo amarillento volviéndose ocráceo anaranjado, laminillas amarillas más claras y estípite marrón rojizo, cubierto por abundantes pelos que le dan un aspecto aterciopelado (Pegler, 1983). Por su hábito collibioide, y pie aterciopelado, cubierto por pelos cortos, marrón rojizos, pertenece a la sección Heimiomyces (Singer, 1965; Miller, 1968; Redhead, 1988). Horak (1979) describe varias especies de Xeromphalina y Heimiomyces (este último considerado como una sección de Xeromphalina) para el Sudeste asiático y Oceanía, describiendo dos especies muy similares, $H$. neovelutipes (Hongo) E. Horak y H. atrofulvus (Stev.) E. Horak, considerándolas a ambas como microespecies geográficas diferentes a $X$. tenuipes. De estas especies descriptas por Horak (1979), H. cespitosus E. Horak, se asemeja mucho al material analizado, con elementos de la pileipellis engrosados y hábito cespitoso, pero se diferencia por presentar esporas más pequeñas.

Distribución. Pantropical (Pegler, 1983; Singer, 1965; Miller, 1968; Redhead, 1988; Yang, 2000). En la Argentina fue citada para las provincias de
Misiones como Heimiomyces tenuipes (Singer, 1950) y como Heliomyces rheicolor (Spegazzini, 1926), Tucumán (Singer \& Digilio, 1952) y Catamarca (Singer, 1965).

\section{Bibliografía}

BURKART, R. N., O. BÁRBARO, R. O. SÁNCHEZ \& D. A. GÓMEZ. 1999. Eco-regiones de la Argentina. Administración de Parques Nacionales - Programa de Desarrollo Institucional Ambiental. Buenos Aires.

CABRERA, A. L. 1994. Regiones fitogeográficas argentinas. Enciclopedia Argentina de Agricultura y Jardinería. Editorial Acme. Buenos Aires.

HORAK, E. 1979. Xeromphalina and Heimiomyces in Indomalaya and Australasia. Sydowia 32: 131-153.

HORAK, E. 1986. Tetrapyrgos Horak (nom, et. Gen. nov.) replacing Pterospora Métrod (1949; nom, preocc). Sydowia 39: 101-103.

INDEX FUNGORUM - Authors of Fungal Names. http://www.indexfungorum.org/ Names/ AuthorsOfFungalNames.asp. (Acceso: Octubre 2012).

LANFIUTTI, A. 1998. Prospección botánica y estado de conservación de la Reserva Natural Estricta Colonia Benitez. Área Integrante del Sistema de la Administración de Parques Nacionales. Informe de la Delegación Técnica Regional N.E.A. Administración de Parques Nacionales.

LARGENT, D. L. 1986. How to identify mushrooms to genus I: macroscopic features. Mad River Press. Eureka.

LODGE, J., J. F. AMMIRATI, T. E. O'DELL, G. M. MUELLER, S. M. HUHNDORF, C. J. WANG, J. N. STOKLAND, J. P. SCHMIT, L. RYVARDEN, P. R. LEACOCK, M. MATA, L. UMAÑA, Q. F. WU \& D. 
CZEDERPILTZ. 2004. Terrestrial and Lignicolous Macrofungi. In G. M. MUELLER, G. F. BILLS, M. S. FOSTER (eds.), Biodiversity of Fungi, Inventory and Monitoring Methods, pp. 127-158. Elsevier Academic Press, San Diego.

MATURO, H. M. \& D. E. PRADO. 2012. Los bosques del Chaco Húmedo, vegetación y fitogeografía. Analisis de vegetación y posición fitogeográfica de la Reserva "El Bagual”" (REB), Formosa, Argentina. Editorial Academia Española. Saarbrücken.

MATURO, H. M., L. J. OAKLEY \& D. E. PRADO. 2005. Vegetación y posición fitogeográfica de la Reserva 'El Bagual'. In: DI GIACOMO, A. G. Y S. F. KRAPOVICKAS (eds.), Historia Natural y Paisaje de la Reserva "El Bagual", Provincia de Formosa, Argentina, pp. 59-73. Aves Argentinas/ AOP, Buenos Aires.

MILLER, O. K. 1968. A revision of the genus Xeromphalina. Mycologia 60: 156-186.

MORELLO, J. \& J. ADAMOLI. 1967. Vegetación y ambiente del nordeste del Chaco argentino (Guia de Viaje, tramo Resistencia - Puerto Pilcomayo). IX Jornadas Botánicas Argentinas, Boletin EEA Colonia Benitez 3: 1-75.

NIVEIRO, N.\& O. POPOFF. 2009. Estudios preliminares de macrohongos de la Reserva Educativa "Colonia Benítez” (Chaco - Argentina): ascomycetes y basidiomycetes. XII Reunión de comunicaciones científicas y tecnológicas; Secretaría General de Ciencia y Técnica-Universidad Nacional del Nordeste (SGCyT-UNNE); In http://www. unne.edu.ar/investigacion/com2009/CB-037.pdf. [Acceso: 30 de Octubre de 2012].

NIVEIRO, N., O. F. POPOFF \& E. ALBERTÓ. 2009a. Estudio preliminar de la diversidad de Agaricales s.l. en la Reserva de Biosfera Yabotí. Bol. Soc. Argent. Bot. 44 (Suplemento): 124-125.

NIVEIRO, N., O. F. POPOFF \& E. O. ALBERTÓ. 2009b. Hongos comestibles silvestres: presencia de especies exóticas de Suillus (Boletales, Basidiomycota) y Lactarius (Russulales, Basidiomycota) asociadas a los cultivos de Pinus elliotti del nordeste argentino. Bonplandia 18 (1):65-71.

NIVEIRO, N., O. F. POPOFF \& E. O. ALBERTÓ. 2010. Contribución al conocimiento de los agaricales s.l. de la Selva Paranaense argentina. Bol. Soc. Argent. Bot. 45: 17-27.

NIVEIRO, N. \& O. F. POPOFF. 2010a. Basidiomycetes en áreas protegidas del Distrito Chaqueño Oriental. I Simposio internacional sobre ecología y diversidad fúngica en zonas áridas y semiáridas en Sudamérica: la diagonal Caatinga-Chaco. Asociación Micológica Carlos Spegazzini IMBIV-CONICET; Córdoba, Córdoba.
PEGLER, D. N. 1983. Agaric flora of the Lesser Antilles. Kew Bull., Addit. Ser. 9: 1-668.

PEGLER, D. N. 1986. Agaric flora of Sri Lanka. Kew Bull., Addit. Ser. 12: 1-519.

POPOFF, O. F. 2000. Novedades sobre Corticioides y Políporos (Aphyllophorales, Basidiomycetes) del nordeste argentino y Paraguay. Tesis Doctoral. Facultad de Ciencias Exactas, Físicas y Naturales, Universidad Nacional de Córdoba, Córdoba.

POPOFF, O. F. \& L. I. FERRARO. 2002. Hongos y Líquenes. En M. M. ARBO \& S. G. TRESSENS (eds.), Flora del Iberá, pp. 381-415. EUDENE, Corrientes.

PRADO, D. E. 1993. What is the Gran Chaco vegetation in South America? I. A review. contribution to the study of flora and vegetation of the Chaco. V. Candollea 48: 145-172.

PRADO, D. E. 2000. Seasonally dry forest of tropical South America: from forgotten ecosystems to a new phytogeographic unit. Edimburgh Jour. Bot. 57: 437-461.

RAITHELHUBER, J. 2004. Nueva flora micológica Argentina. Mycosur. Stuttgart.

REDHEAD, S.A. 1988. Notes on the genus Xeromphalina (Agaricales, Xerulaceae) in Canada: biogeography, nomenclature, taxonomy. Can. J. Bot. 66: 497-507.

SINGER, R. \& A. P. L. DIGILIO. 1952 [1951]. Pródromo de la flora agaricina argentina. Lilloa 25: 6-461.

SINGER, R. 1950. Type studies on Basidiomycetes IV. Lilloa 23: 147-246.

SINGER, R. 1965. Monograph of South America Basidyomycetes, especially those of the east slope of the Andes and Brazil X. Xeromphalina. Bol. Soc. Argent. Bot. 10: 302-310.

SINGER, R. 1973. The genera Marasmiellus, Crepidotus and Simocybe in the Neotropics. Beih. Nova Hedwigia 44: 1-517.

SINGER, R. 1986. The Agaricales in modern taxonomy. 4th ed. Koeltz Scientific Books. Koenigstein.

SPEGAZZINI, C. 1899 [1898]. Fungi argentini novi v. critici. Anales Mus. Nac. Buenos Aires 6: 6-365.

SPEGAZZINI, C. 1919. Los hongos del Tucumán. Primera Reunión Nacional de la Soc. Arg. de Cs. Naturales: Tucumán, 254-274.

SPEGAZZINI, C. 1926. Observaciones y adiciones a la micología argentina. Bol. Acad. Ci. (Córdoba) 28: 267-351.

THIERS, B. 2012. Index Herbariorum: A global directory of public herbaria and associated staff. New York Botanical Garden's Virtual Herbarium. http:// sweetgum.nybg.org/ih/. [Acceso: Octubre 2012].

WEBSTER, J. \& R. W. S. WEBER. 2007. Introduction to Fungi. $3^{\text {rd }}$ ed. Cambridge University Press. New York.

WRIGHT, J. E. \& E. WRIGHT. 2005. Checklist of the 
Bol. Soc. Argent. Bot. 48 (3-4) 2013

mycobiota of Iguazú National Park (Misiones, Argentina). Bol. Soc. Argent. Bot. 40: 23-44.

WRIGHT, J. E. \& E. ALBERTÓ. 2002. Hongos. Guía de la región Pampeana. I. Hongos con laminillas. L.O.L.A. Buenos Aires.

YANG, Z. L. 2000. Notes on five common but little known higher Basidiomycetes from tropical Yunnan, China. Mycotaxon 74: 45-56.

Recibido el 7 de noviembre de 2012, aceptado el 27 de febrero de 2013. 